\title{
ROAD ACCIDENTS AND THE FAMILY DOCTOR
}

\section{Spinal Injuries and Fractures}

\author{
J. ASHWORTH,* F.R.C.S.
}

British Medical fournal, 1969, 4, 414-415

About three hundred cases of damage to the spinal cord are reported each year, and most of these are caused by an accident in a motor vehicle. Moreover, such accidents are becoming more frequent. Probably six to seven times this number of spinal injuries occur without neurological damage.

A typical vertebra is made up of two columns; the posterior one comprises the articulations supported by the ligamenta flava and the interspinous and supraspinous ligaments between the spinous processes, and the anterior one consists of the body and adjacent discs and the anterior and posterior longitudinal ligaments.

\section{Classification of Spinal Injuries}

Injuries to the spine may be classified as stable or unstable, and it is invariably in the unstable injuries that damage to the cord occurs. For a spine to become unstable both anterior and posterior columns must be disrupted. The usual type of force occurring in a road accident is that of flexion, which puts the posterior ligaments under tension. Nevertheless, as these ligaments are relatively strong in comparison to the cancellous bone of the vertebral bodies, they often remain intact and a simple wedge fracture of the body occurs. This is a stable type of injury, in which neurological damage is rare, and only with increased force does the posterior column give way, thus resulting in displacement and the likelihood of cord compression.

Sometimes the nature of the force is a vertical compression, which tends to affect specific areas. The spine may be thought of as having two mobile areas in the cervical and lumbar regions and a third in the thoracic region; the latter is formed inte a rigid box by the ribs and sternum. The mobile areas are normally lordotic, but become straight on flexion, and the vertical force applied in this position causes a burst fracture of the vertebral body. This fracture, though stable, may cause cord damage, because the bursting nature of the injury may result in fragments being pushed posteriorly and into the spinal canal.

A rotational force may occur, and this is usually associated with a flexion force resulting in rupture of the ligaments, which leads to an unstable type of injury. This type of force can cause different injuries to the three regions of the spine.

In the cervical region the articular facets are virtualiy horizontal. Hence a shearing force resulting in rupture of the ligaments of the anterior and posterior columns easily produces a forward dislocation of the upper on the lower vertebra. It is extremely unstable, so that forward and backward movements of the upper segment are possible. Hence, if cord damage does not occur at the time of the accident, this is still possible during extrication of the person from the vehicle or during transportation to hospital, particularly in an unconscious person.

Injuries in the thoracic region are rather different. If the shearing force causes ligamentous damage-and therefore in theory an unstable injury-fractures are still usually stable because the solid cage formed by the ribs and sternum holds the fractures together.

* Consultant Orthopaedic Surgeon, Darlington and Nurthallerton Hospitals, Yorkshire.
In the lower thoracic and lumbar regions the articular facets are almost vertical. A shearing force here causes not only a dislocation, but also a fracture of one or both facets, and a compression fracture of the vertebral body. These injuries are unstable, and are almost always complicated by spinal cord damage, which occurs at the time of impact.

Extension injuries are rare, but may occur in the cervical region. In an elderly person such injuries may cause cord damage even though an $x$-ray film does not show any displacement. These extension injuries are theoretically stable and the cord damage is due to infolding either of the ligamentum flavum or of the posterior longitudinal ligament.
$\underline{A}$

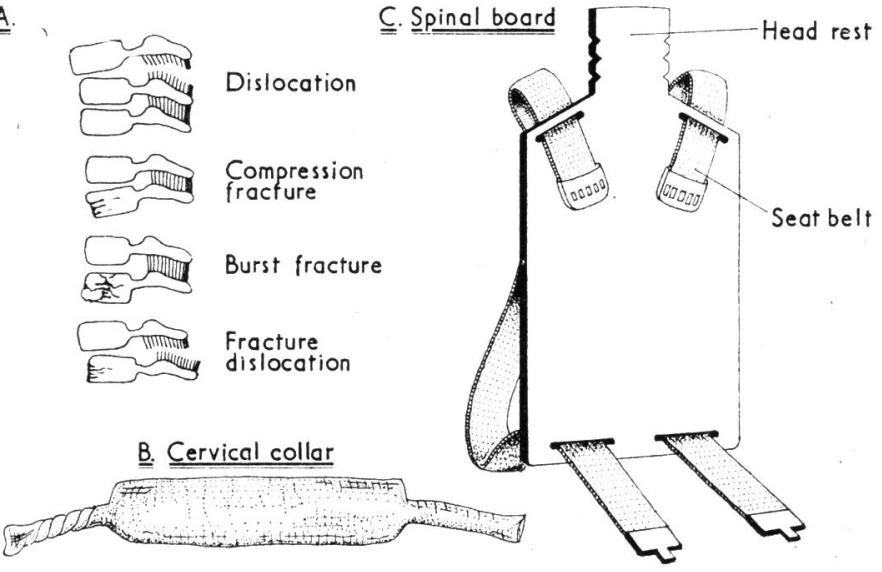

Diagnosis
The diagnosis of a spinal injury at the roadside is a vastly different problem from diagnosis with a well-equipped accident unit. If one can examine the patient the presence of a palpable gap between two spinous processes suggests an unstable fracture, as it denotes tearing of the supraspinous and interspinous ligaments with opening up of the space between the adjacent processes. Tenderness over an area of the spine may imply bony injury, and if this is found on early examination at the roadside it must be taken as diagnostic of bone injury. If the person is conscious he may complain of pain over a segment of the spine. Neurological damage may be indicated if he is unable to move one or more limbs or if he has sensory disturbances in either the arms or the legs. The presence of an abrasion on the top of the skull may denote injury from a vertical force, thus causing a possible burst fracture of the cervical spine.

\section{Treatment}

With the exception of injuries in the cervical region, any neurological damage usually occurs at the time of impact. Hence there is nothing that can be done at the site of the accident that will have any effect on the ultimate prognosis. In the cervical region, however, the facets are inclined in such a way that recurrence of a dislocation may take place during. 
movement of a person from the involved vehicle or during transportation to hospital. Treatment at the roadside may therefore have a considerable effect on the prognosis of people in this group.

As many of these people with injuries to the cervical spine may also have severe head injuries a large proportion of them will either be unconscious or unable to give any clear indication of any disturbance of motor or sensory function in the limbs. Injury to the cervical spine therefore often has to be assumed. This means that many people will be treated unnecessarily, in view of the difficulty of making an accurate diagnosis at the roadside, of ten in appalling conditions. Overtreatment at this stage, however, is never contraindicated ; therefore if there is any suspicion of injury, particularly to the cervical region, the affected part should be immobilized.

\section{Immobilization}

Immobilization of the cervical spine is a relatively simple and effective procedure. As most injuries in this region are due to a flexion force producing either a compression fracture or a dislocation, with the possibility of concurrent neurological damage, prevention of further flexion movements is all that is required to avoid a recurrent subluxation or dislocation. This may be done adequately with any form of cervical collar which fits underneath the jaw and thus prevents it from sagging forward on to the sternum.

Cervical Collar.-A home-made collar, consisting of a length of stiff cardboard (12 in. $\times 3$ in. ; $30 \mathrm{~cm} . \times 7 \cdot 5 \mathrm{~cm}$.) covered with foam rubber, felt, or wool for comfort and enclosed in a piece of tubular gauze, which is used to fasten the collar round the neck, is simple and effective and can be carried by all general practitioners in a minimum of storage space. The piece of cardboard, which should be long enough to run under the angle of the jaw to the occiput, will also prevent hyperextension; this may be particularly useful in elderly people who sustain an extension type of injury. Moreover, the collar will serve to hold forward the angle of the jaw, and therefore is extremely effective in preventing the tongue from falling backwards and causing airway obstruction in an unconscious patient.

The collar can be applied to the patient's neck while he is still in the vehicle; it can be fitted in any position and then rotated round into the correct site. With the collar in situ the injured person may then be removed from the vehicle in much greater safety, particularly if he does have an unstable injury to his cervical spine. As the collar does not have to be pulled tight, there is no danger of impeding venous return or of causing any pressure on the neck at all. Once the patient has been extricated from the vehicle he may be transported in whatever position is most suitable in view of any other injuries.

Though in a motor-car accident injuries to the thoracic spine are seldom complicated by cord damage, they do occur frequently in motor-cycle accidents. In the latter case the shearing force is greater and a fracture dislozation may occur with cord damage. This damage occurs at the time of the accident and any form of immobilization is probably of value only in making transportation easier, the patient more comfortable, or in enabling him to be put into the required position for the treatment of other injuries.

Similarly any cord damage which complicates injuries to the lower thoracic and lumbar spine will usually have occurred at the time of impact and not after. Immobilization of injuries to this region is recommended for the same reasons as those mentioned in relation to injuries of the thoracic spine.

Spinal Board.-It is very often much easier to remove a person from a vehicle, to transport him in reasonable comfort, and to treat other injuries if immobilization of the injured part is carried out. For this purpose a modified form of spinal board is advocated, which is simple to apply to the spinal injury and provides a surprising degree of immobilization. The easiest and most comfortable position for the patient when being moved is either on his side or in an inclined position (if other injuries necessitate this) once the injured spine has been immobilized.

All that is required to produce a spinal board is a piece of five-ply board, a saw, and a pair of safety belts, and for this reason it costs very little. The only other requirement is either a broad triangular bandage or a large handkerchief to hold the skull against the upper part of the board.

Only in injuries of the cervical spine will treatment have any appreciable effect on the patient's prognosis, but, as it is in this region that the increase in incidence of spinal injuries is most pronounced, a worthwhile decrease in morbidity may be achieved. In addition, however, as so many of these people suffer from multiple injuries, immobilization of the thoracic and lumbar regions may help problems of resuscitation or airway control to be overcome more easily, and to allow transportation of the patient in greater comfort.

\section{ANY QUESTIONS ?}

We publish below a selection of questions and answers of general interest.

\section{Aerosol as Humidifier}

Q.-Has non-specific aerosol therapy any important role in the treatment of asthma or chronic bronchitis?

A.-An aerosol of water or saline produced by an efficient nebulizer or mist tent capable of delivering a dry mist with a particle size down to 1 micron is effective in humidifying inspired gas. The addition of water vapour is thought to aid expectoration of bronchial mucus.

Aerosol therapy is generally regarded as beneficial in aiding the expectoration of viscoid bronchial secretions in several broncho-pulmonary diseases, including severe asthma, exacerbations of chronic obstructive bronchitis, acute bronchiolitis of infants, and cystic fibrosis.
Ultrasonic nebulizers can deliver large volumes of water to the lung. Their role in therapy remains to be evaluated.

\section{Masking Scars}

Q.-Is there any application which can be used to mask noticeable scars such as a thyroidectomy scar?

A.-There are a number of skin-masking creams on the market. They are more effective when the scar or blemish is flat. When the surface is uneven the cosmetic result may not be so good. Patients may be referred to the manufacturing company, where they can be shown how to use the covering cream most effectively.

\section{Senile Mental Deterioration}

Q.-Is there any drug which increases the blood supply to the brain in old age and stimulates the mental faculties?

A.-There are several drugs which are believed to cause cerebral vasodilatation. The action of cyclandelate has been investigated by various methods. Luisada et al., using rheoencephalography in a group of 26 old people whose average age was 80.4 years, demonstrated vasodilatation when measurements were carried out two and six weeks after the start of treatment with cyclandelate $200 \mathrm{mg}$. four times daily.

O'Brien and Veall $^{2}$ measured cortical perfusion by timing the clearance of inhaled radioactive xenon in nine patients with cerebrovascular disease. Each patient acted as his own control, and clearance rates from each cerebral hemisphere were measured 